University of Nebraska - Lincoln

DigitalCommons@University of Nebraska - Lincoln

May 1991

\title{
Probing the metal-nonmetal transition in thin metal overlayers using resonant photoemission
}

\author{
Peter A. Dowben \\ Syracuse University and University of Nebraska-Lincoln, pdowben@unl.edu \\ D. LaGraffe \\ Syracuse University \\ Dongqi Li \\ Syracuse University \\ G. Vidali \\ Syracuse University \\ L. Zhang \\ Syracuse University \\ See next page for additional authors
}

Follow this and additional works at: https://digitalcommons.unl.edu/physicsdowben

Part of the Physics Commons

Dowben, Peter A.; LaGraffe, D.; Li, Dongqi; Vidali, G.; Zhang, L.; Dottl, L.; and Onellion, M., "Probing the metal-nonmetal transition in thin metal overlayers using resonant photoemission" (1991). Peter Dowben Publications. 63.

https://digitalcommons.unl.edu/physicsdowben/63

This Article is brought to you for free and open access by the Research Papers in Physics and Astronomy at DigitalCommons@University of Nebraska - Lincoln. It has been accepted for inclusion in Peter Dowben Publications by an authorized administrator of DigitalCommons@University of Nebraska - Lincoln. 


\section{Authors}

Peter A. Dowben, D. LaGraffe, Dongqi Li, G. Vidali, L. Zhang, L. Dottl, and M. Onellion 


\title{
Probing the metal-nonmetal transition in thin metal overlayers using resonant photoemission
}

\author{
P. A. Dowben, ${ }^{*}$ D. LaGraffe, Dongqi Li, G. Vidali, and L. Zhang \\ Department of Physics, Syracuse University, Syracuse, New York 13244-1130 \\ L. Dottl and M. Onellion \\ Department of Physics, University of Wisconsin, Madison, Wisconsin 53706
}

(Received 19 December 1990)

\begin{abstract}
We have studied one and two monolayers of barium on $\mathrm{Ni}(111)$ and of mercury on $\mathrm{Cu}(100)$. Using resonant photoemission, we have found core excited electrons become delocalized with increasing barium coverage. Similarly, upon formation of the mercury bilayer (as determined by lowenergy electron diffraction and by atom-beam scattering), there is a substantial increase in the screening of the photohole. A transition of the electronic structure akin to a metal-nonmetal (metal-insulator) transition is apparent in these final-state effects. The band structure for $\mathrm{Hg}$ is similar to the band structure expected for a free-standing film with a free-electron $s d$ band. The delocalization of the core excited electrons resembles the exciton unbinding that occurs at the metalnonmetal Mott transition.
\end{abstract}

\section{INTRODUCTION}

The metal-to-nonmetal transition in the divalent metals, in particular mercury, ${ }^{1-6}$ and to a considerably lesser extent barium, ${ }^{7}$ has been the subject of a large number of studies. In general, a metal-nonmetal transition in the one-electron picture occurs when the filled $6 s$ band hybridizes and overlaps the unfilled $6 p$ band. This is the Wilson-type metal-nonmetal transition and is often ascribed to the metal-insulator transitions in the divalent metals, whereas the monovalent metals should exhibit the Mott-type metal-nonmetal transition. ${ }^{1,2}$ Because of band splitting, the metal-insulator transition that occurs at low densities in liquid $\mathrm{Hg}$ is considered to be a Wilson-type transition. ${ }^{1}$ The Wilson model, in its simpliest form, requires that the phase transition be second order, ${ }^{1}$ and does not allow for any " $s$-like" or localized hybrid states at the Fermi level of mercury in the nonmetallic phases. ${ }^{3,8,9}$ These predictions from the Wilson model fail to agree with experimental results obtained for bulk mercury ${ }^{1,10-16}$ in part because the transition appears to be first order ${ }^{1,11,12}$ or at least not a "simple" second-order phase transition. The Wilson model also is not consistent $^{3}$ with optical-reflectivity data. ${ }^{13-16}$ Experimental results for bulk mercury have been found to be consistent with a Mott-type metal-nonmetal transition. ${ }^{3,12}$

The Mott-type transition is essentially a many-bodytype transition that is typically applied to metal-nonmetal transitions in the monovalent metals. In the Mott-type metal-nonmetal transition, the nonmetal is an excitonic insulator, ${ }^{1,17,18}$ and the excitons unbind at the metalnonmetal transition. ${ }^{3}$ Free carriers will affect the formation of excitons by either screening the Coulomb field between the electron-hole pair or shorten the lifetime of the exciton. ${ }^{18}$ In resonant photoemission, a core exciton is formed. ${ }^{19-24}$ A core electron is excited to an unoccupied state near the Fermi energy, forming a transient excited state. The final state is identical, with the direct photo- emission process thus leading to a resonant enhancement of valence-band photoemission features at the characteristic photon energy necessary to form the excited state. Changes in the density of conduction electrons should influence the core exciton or the photohole, thus resulting in changes of the partial photoemission cross sections of various initial states. In a preliminary $\operatorname{paper}^{25}$ we have demonstrated that, indeed, resonant photoemission can be used to probe the metal-nonmetal transition in such divalent metals as barium.

For divalent-metal overlayers adsorbed on metal substrates, it is often impractical to observe changes in the electronic structure of the overlayer directly by photoemission. This is particularly true of nickel substrates because of the large density of states near the Fermi energy. Resonant photoemission, however, can be used to probe the electronic structure of some states near the Fermi level for high- $Z$-metal overlayers. ${ }^{25,26}$ In this study, we have probed the changes in electronic structure of barium overlayers on $\mathrm{Ni}(111)$ and of mercury overlayers on $\mathrm{Cu}(100)$ by resonant photoemission and photoemission across the shallow $5 p$ core levels of barium and the shallow $5 d$ core levels of mercury.

The Ni(111) substrate was chosen for the barium study because studies of mercury monolayers on $\mathrm{Ni}(111)$ have been observed to undergo structural phase transitions accompanied by changes in the electronic band structure. ${ }^{27-29}$ It has been postulated that the transition is driven by electronic-structure effects related to the metal-nonmetal transition. ${ }^{27,28}$ Unlike mercury, ${ }^{30}$ the electronic structure of barium overlayers has not been investigated, apart from studies of the giant $4 d-4 f$ resonance ${ }^{31-35}$ and studies of $\mathrm{BaO}$ overlayers. ${ }^{36-38}$

$\mathrm{Cu}(100)$ was chosen as the substrate for the mercury overlayers because at normal emission $\left(\bar{\Gamma}\right.$ and $\left.\mathbf{k}_{11}=0\right)$, the $\mathrm{Cu}(100)$ exhibits a negligible density of states near the Fermi energy, for photon energies between 30 and $70 \mathrm{eV}$. We have also undertaken a number of studies addressing 
the adlayer structure of mercury overlayers on $\mathrm{Cu}(100)$ by both low-energy electron diffraction (LEED) and atom-beam scattering (ABS). ${ }^{39-41}$ In this study, we have concentrated our attention on $\mathrm{Hg}$ overlayers which adopt a square-lattice structure at submonolayer coverages. ${ }^{30}$ Such overlayers are not expected to exhibit an abrupt metal-nonmetal transition with changing lattice constant, ${ }^{5}$ though Mott $^{42}$ demonstrated that a cubic lattice of one-electron atoms could be an insulator at sufficiently large lattice spacings as a result of decreasing atomicorbital overlapping. Experimental studies of $\mathrm{Hg}$ monolayers arranged in a square lattice have demonstrated that the formation of the two-dimensional band structure is very sensitive to the size of the lattice constant. ${ }^{30}$ As suggested by Jansen, Freeman, Weinert, and Wimmer, ${ }^{5}$ adsorption of mercury films on a substrate that interacts only weakly with the adlayer makes possible the study of adlayers with a wide range of lattice constants ${ }^{30,39}$ without resorting to high temperatures or high pressures.

\section{EXPERIMENT}

The photoemission system used for these studies has been described in detail elsewhere, ${ }^{26}$ and all photoemission spectra were taken with a hemispherical electronenergy analyzer with an angular resolution of $\pm 1.5^{\circ}$. The photon-energy source employed for these photoemission studies was the 1-GeV Alladin ring of the Synchrotron Radiation Center at Stoughton, Wisconsin. The light was dispersed with either a 6-m or a 3-m toroidal grating monochromator (TGM). The combined resolution of the electron-energy analyzer and the monochromator was 150-200 meV. The atom-beam-scattering studies and the studies undertaken to determine the structural phase diagram of mercury overlayers on $\mathrm{Cu}(100)$ were carried out in a separate vacuum system, as described in detail elsewhere. ${ }^{39,41}$

All the photoemission studies in this work were undertaken at normal emission unless otherwise indicated. The binding energies of the photoemission features are referenced to the Fermi energy of the clean $\mathrm{Cu}(100)$ or $\mathrm{Ni}(111)$ substrate. The partial photoemission cross sections or constant-initial-state (CIS) spectra were carried out as described elsewhere. ${ }^{25,26}$ The kinetic energy and photon energies were changed together, so as to determine the relative photoemission intensity of a given initial state (say, for example, the features near the Fermi energy). The resolution of both the monochromator and the electron-energy analyzer were degraded to more than 500 meV. The CIS spectra were normalized for photon flux through the monochromators using the current from a gold diode at the exit of the monochromator. The light incidence angle for most of this work was $60^{\circ}$ off the normal (principally $p$-polarized light) unless otherwise indicated.

The $\mathrm{Ni}(111), \mathrm{Ag}(100), \mathrm{Cu}_{3} \mathrm{Ag}(100)$, and $\mathrm{Cu}(100)$ crystal substrates were all cleaned by repeated annealing and $\mathrm{Ar}^{+}$-ion sputtering treatments. $\mathrm{Hg}$ adsorption on the $\mathrm{Ag}(100), \mathrm{Cu}_{3} \mathrm{Au}(100)$ surface was undertaken with the substrates cooled to $80-100 \mathrm{~K}$, as described elsewhere, ${ }^{43}$ while $\mathrm{Hg}$ adsorption on $\mathrm{Cu}(100)$ was undertaken at $220 \mathrm{~K}$ as described in studies undertaken to characterize the structural phases. ${ }^{39}$ The temperature of adsorption ensured that the $\mathrm{Hg}$ overlayers would grow epitaxially in the $1 \times 1$ structure for the first two monolayers on $\mathrm{Ag}(100)$ and $\mathrm{Cu}_{3} \mathrm{Au}(100),{ }^{43}$ while for $\mathrm{Hg}$ on $\mathrm{Cu}(100)$ the overlayer would first form $c(2 \times 2)$, then a $c(4 \times 4)$ phase, followed by adsorption into a second layer, with the adatoms in the second layer adsorbing first into the highcoordination sites. ${ }^{40}$ Coverages were determined from a combination of angle-resolved photoemission and Auger electron studies as described previously. ${ }^{43}$ For all of the $\mathrm{Hg}$ overlayers, the structures have been identified by diffraction techniques (LEED and ABS), and the overlayer growth mode is known to be layer by layer for at least the first two mercury layers, as shown by ABS, Auger electron spectroscopy, and angle-resolved photoemission.

The barium was deposited from a commercial barium dispenser cathode (SAES). Previous experiments showed that the films were uncontaminated within the sensitivity of our Auger electron spectrometer, but photoemission studies indicated that the barium overlayers oxidized or adsorbed contaminants even at ambient pressures less than $2 \times 10^{-10}$ Torr. This limited the number of studies that could be reliably undertaken for any one deposited film. In general, to provide assurance that contamination (even small amounts) did not influence our results, measurements were completed within 20-30 min after evaporation. Some oxygen contamination is evident in the photoemission spectra taken within 60-120 min after evaporation, with background pressures at $2 \times 10^{-10}$ Torr or less. The photoemission feature at $4 \mathrm{eV}$ binding energy is believed to be at least partially due to contamination. ${ }^{34,36}$ The coverages of the barium overlayers have been determined using a commercial thin-film monitor, but the stated thickness must be considered only an approximate measure of the barium coverage, and substantial errors in the absolute coverages are possible, though for convenience we have referred to these nominal coverages. The relative coverages of each of the barium overlayers are very accurate and are in error by no more than $10 \%$, as determined by the photoemission studies of the barium core levels. Unlike mercury, the bariumoverlayer growth mode and overlayer structurs are not known, although there is some evidence that barium will grow layer by layer for at least the first monolayer. ${ }^{44}$

\section{IDENTIFICATION OF THE EQUILIBRIUM PHASES OF Hg ADSORBED ON Cu(100)}

The adsorption of mercury on clean $\mathrm{Cu}(100)$ is very sensitive to small amounts of contamination, as determined by studies of the isosteric heat of adsorption ${ }^{39}$ and the heat of desorption. ${ }^{45}$ With a clean surface, the adsorption of mercury is possible over a very wide range in substrate temperature. ${ }^{39,40}$ Relative changes in the ABS specular beam scattering intensity off the $\mathrm{Cu}(100)$ can be associated with the adsorption of $\mathrm{Hg}$, as seen in Fig. 1 . With adsorption of $\mathrm{Hg}$ on $\mathrm{Cu}(100)$ at $200 \mathrm{~K}$, the ABS intensity is seen to decrease until about 4.5 langumirs (L) $\mathrm{Hg}$ exposure. With continued exposure, the ABS intensi- 
ty is seen to increase, though the rate of this increase abruptly changes at an $\mathrm{Hg}$ exposure of about 8 langmuirs (L), as seen in Fig. 1.

Two different low-energy electron-diffraction (LEED) patterns have been identified following $\mathrm{Hg}$ adsorption between 180 and $330 \mathrm{~K}$. These two LEED patterns will be identified, for convenience, by their coincidence overlayer net structures (as discussed in detail elsewhere $\left.{ }^{39}\right), c(2 \times 2)$ and $c(4 \times 4)$. The structures are shown schematically in Fig. 2. The first-order diffraction beams of both structures have been observed in ABS at $45^{\circ}$ off and $\pm 18.4^{\circ}$ off the $\langle 1 \overline{1} 0\rangle$ azimuth for the $c(2 \times 2)$ and the $c(4 \times 4)$, respectively, as seen in Fig. 2, using a helium beam cooled to $97 \mathrm{~K}$. By combining atom-beam scattering, which probes only the topmost layer, with the results obtained from LEED, ${ }^{39}$ the $c(2 \times 2)$ is identified as an $\mathrm{Hg}$ structure with a maximum coverage of $0.5 \mathrm{Hg}$ atoms per surface copper atom and a lattice constant of $3.62 \AA$, and the $c(4 \times 4)$ has a maximum coverage of $0.62 \mathrm{Hg}$ atoms per surface copper atoms and a lattice constant of $3.22 \AA$.

The $c(4 \times 4)$ is a high-density structure that along the $\langle 010\rangle$ azimuth exhibits a superlattice diffraction pattern in atom-beam scattering, as seen in Fig. 3. Careful analysis of the superlattice diffraction shows that some $\mathrm{Hg}$ atoms lie in sites coincident with lattice net of the $\mathrm{Cu}(100)$ surface. This results in a slight buckling of the overlayer lattice. From the ABS results, the buckling appears to be about a $0.15-\AA$ vertical displacement of the coincident overlayer $\mathrm{Hg}$ atoms. Schematically, this rumpled surface is shown in Fig. 4.

We used LEED to obtain the phase diagram shown in Fig. 5. The symbols represent the maximum intensity of the LEED patterns at that given temperature. For the range of temperatures presented, no annealing of the overlayer was necessary to obtain the maximum intensity. In the inset of Fig. 5, we present ABS diffraction data with the symbols representing the maximum of the

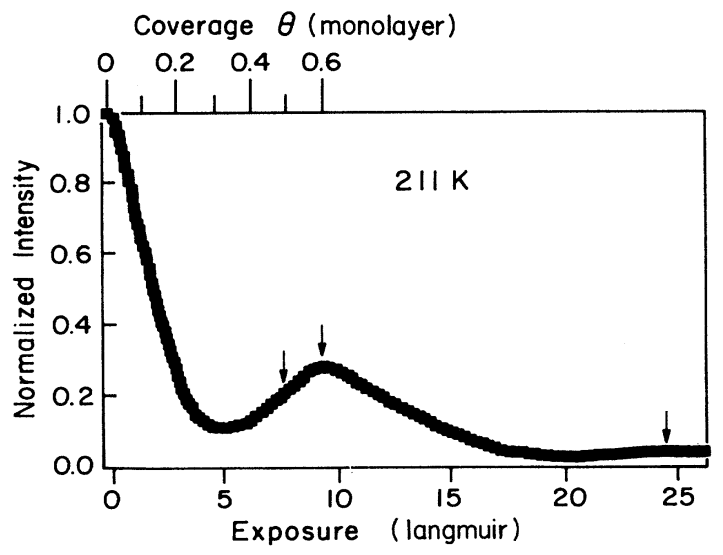

FIG. 1. The relative atom-beam-scattering intensity of the specular beam off of $\mathrm{Cu}(100)$ as a function of exposure to $\mathrm{Hg}$ at $1 \times 10^{-8}$ Torr. The helium beam has an incident energy of 63 $\mathrm{meV}$. The isothermal ABS adsorption curve was taken at 210 K. The arrows indicate the phase transitions from $c(2 \times 2)$ to $c(4 \times 4)$ (left) and from $c(4 \times 4)$ to second layer (middle) and completion of the second layer (right).

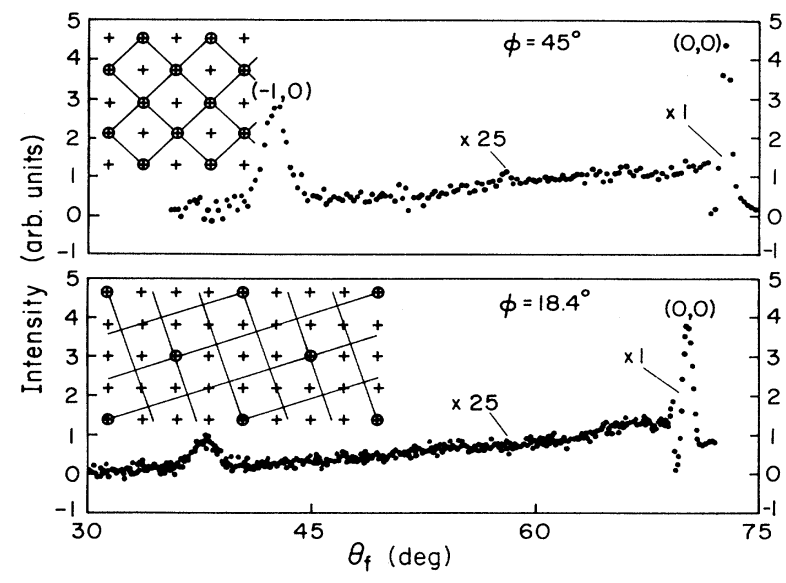

FIG. 2. Helium-atom-diffraction scans from the $c(2 \times 2)$ (top) and the $c(4 \times 4)$ (bottom) $\mathbf{H g}$ overlayers. The helium-atom incidence angle is $\theta_{i}=70^{\circ}$ (top) and $\theta_{i}=71.2^{\circ}$ (bottom). The helium-atom incident energy is $21 \mathrm{meV}$. The real space structures are shown in the insets, where the copper substrate lattice is shown as crosses and the $\mathrm{Hg}$ atoms are shown with circles if they are in registry with the substrate; otherwise they are at the intersection of the lines. No assumption as to the binding site is made in these schematic figures. The details are outlined in Ref. 39.

diffraction peaks. In a ABS typical run, the detector is set at the angular position of a Bragg-diffraction peak pertinent to the structure of the overlayer to be observed. Thermal-desorption studies ${ }^{45}$ indicate that the coverage range for the coexistence of the $c(2 \times 2)$ and the $c(4 \times 4)$ structures is far greater than is required by Fig. 5, for temperatures well below those necessary to overcome the necessary surface activation barriers (i.e., at temperatures in the vicinity of $200 \mathrm{~K}$ ). The structures at these temperatures nonetheless continue to be the equilibrium structures [as opposed to the nonequilibrium structures observed with adsorption at substrate temperatures well below $180 \mathrm{~K}$ (Refs. 39 and 40)].

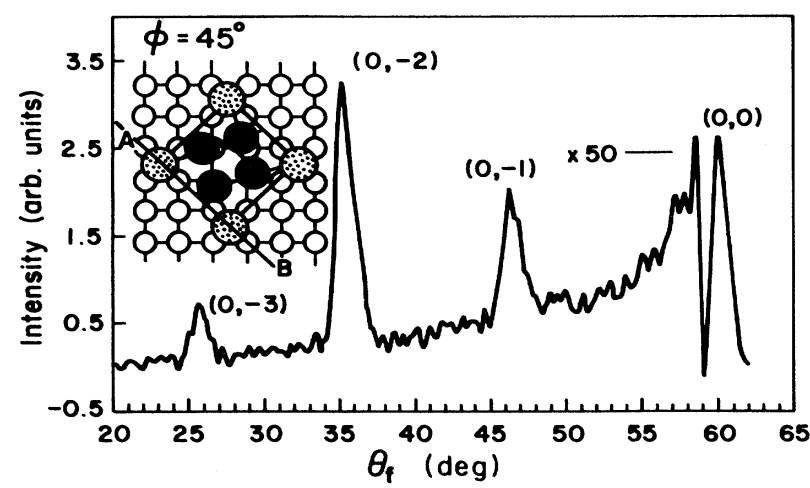

FIG. 3. Helium-atom diffraction of the $c(4 \times 4)$ along the $\langle 010\rangle$ azimuth exhibiting the superlattice diffraction. The helium-atom-beam incident energy is $21 \mathrm{meV}$. The diffracted peaks are indexed with respect to the $c(4 \times 4)$ overlayer (Ref. 40). 


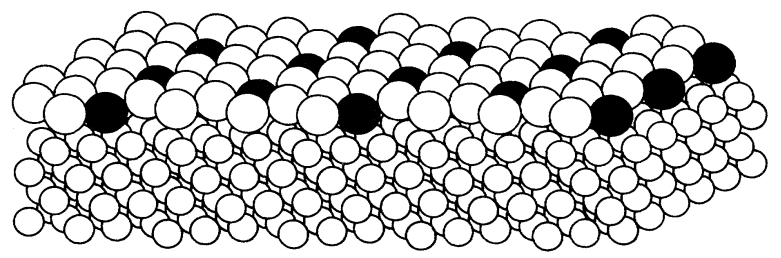

FIG. 4. A schematic representation of the $c(4 \times 4)$ overlayer on $\mathrm{Cu}(100)$ showing the effect of rumpling. The small circles represent the copper atoms.

The small decline in the ABS intensity at $\mathrm{Hg}$ exposures greater than about 10 langmuirs for the adsorption curve shown in Fig. 1 is due to the increasing disorder in the overlayer as a result of atoms adsorbing on top of the first $\mathrm{Hg}$ layer, which occurs upon completion of the $c(4 \times 4)$ overlayer. Atom-beam-scattering data, shown in Fig. 6, indicate that the second $\mathrm{Hg}$ layer adsorbs in registry with the first layer. The initial adsorption begins with the occupation of high-coordination sites created by the $0.15-\AA$ vertical displacement, as shown schematically in Fig. 6. Thus adsorption of an ordered bilayer of $\mathrm{Hg}$ on $\mathrm{Cu}(100)$ is possible.

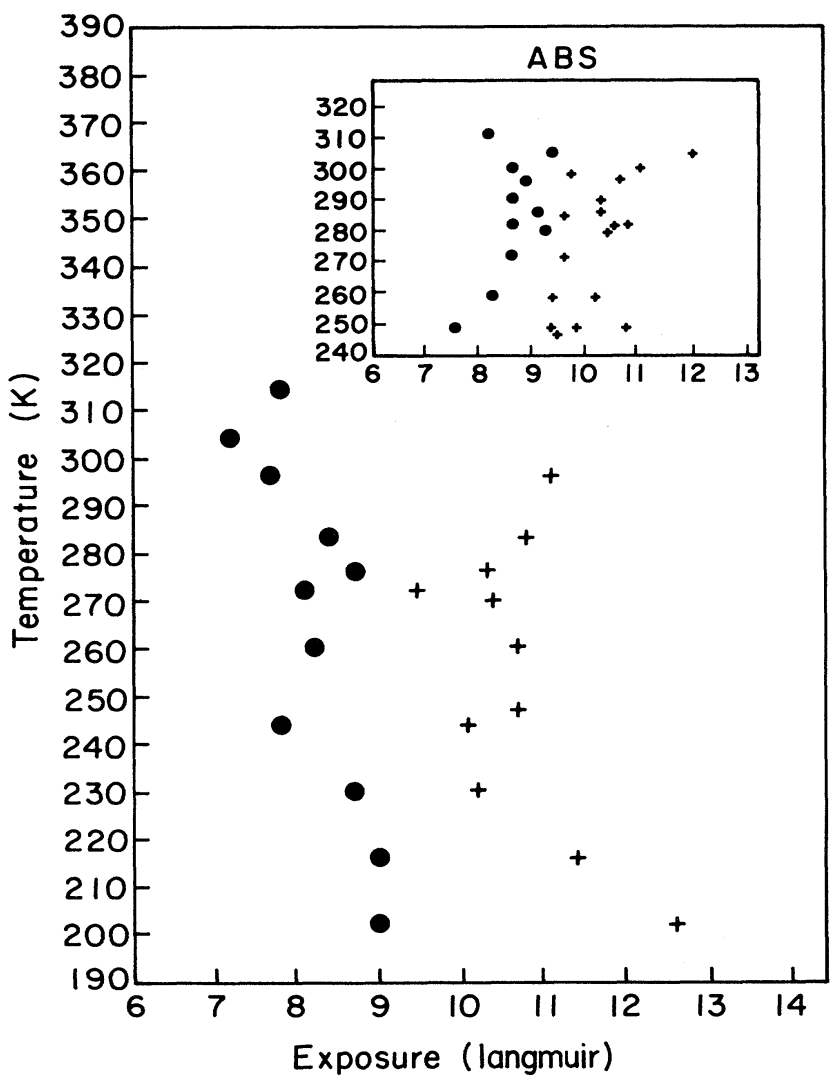

FIG. 5. The phase diagram obtained using LEED and ABS (inset). The symbols indicate the maximum diffraction intensities of the $c(2 \times 2)(0)$ and the $c(4 \times 4)(+)$ phases (Ref. 39).

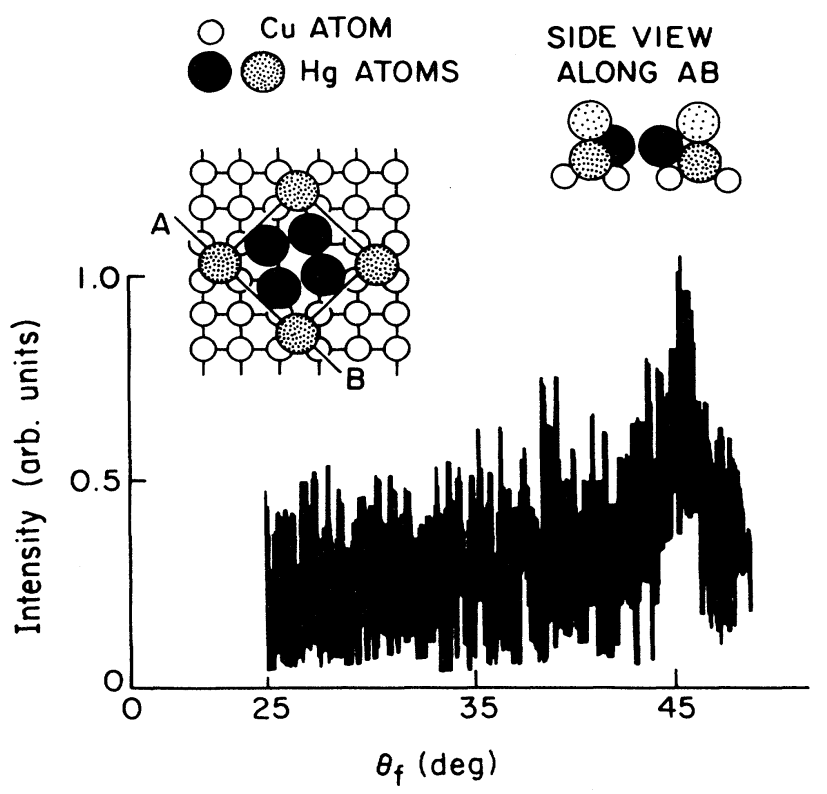

FIG. 6. ABS diffraction from the second layer showing that the second $\mathrm{Hg}$ layer is in registry with the first layer. Scattering is along the $\langle 010\rangle$ direction as indicated. A schematical representation of the bilayer is shown. The second layer adopts a regular $c(4 \times 4)$ lattice as shown.

\section{THE ELECTRONIC STRUCTURE OF Hg OVERLAYERS ON Cu(100)}

The appearance of the Hg-induced photoemission features and the attenuation of the $\mathrm{Cu}(100)$ photoemission features for adsorption at $220 \mathrm{~K}$ is shown in Fig. 7. The most prominent features at coverages up to the completion of the $c(4 \times 4)$ (i.e., less than $10-11 \mathrm{~L}$ ) are the spin-orbit-split $5 d_{3 / 2}$ and $5 d_{5 / 2} \mathrm{Hg}$ features at $9.73 \pm 0.1$ and $7.86 \pm 0.2 \mathrm{eV}$, respectively, at normal emission. The full width at half maximum for these two features are $0.48 \mathrm{eV}\left(5 d_{3 / 2}\right)$ and $0.65\left(5 d_{5 / 2}\right)$. Somewhat in conflict with previous reports, ${ }^{46}$ subtraction of the clean $\mathrm{Cu}(100)$ density of states, even when substantial weight is given to the clean $\mathrm{Cu}(100)$ photoemission energy-distribution curves (as seen in Fig. 7), indicates that there is a broad $\mathrm{Hg}$-induced feature at a binding energy of $4.7 \pm 0.3 \mathrm{eV}$, a narrow $\mathrm{Hg}$-induced feature with a binding energy of $2.2 \pm 0.1 \mathrm{eV}$, and increasing density of states near the Fermi energy. There is no evidence that any of these $\mathbf{H g}$ induced features disperse in binding energy either as a function of photon energy $(20 \mathrm{eV} \leq h v \leq 110 \mathrm{eV})$ or as a function of emission angle. For $\mathrm{Hg}$ coverages up to the completion of the $c(4 \times 4)$, the emission-angle dependence of the cross section and branching ratios of the $\mathrm{Hg}$ $5 d$ levels is found to be very slight. ${ }^{47}$

For $\mathrm{Hg}$ exposures from 16 to $22 \mathrm{~L}$ onto the $\mathrm{Cu}(100)$ sample at $220 \mathrm{~K}$, we adsorb a second layer of $\mathrm{Hg}$ as determined by the attenuation of the $\mathrm{Cu}(100)$ substrate photoemission features, ${ }^{46}$ and from the ABS scattering intensities (described in the previous section). The two 


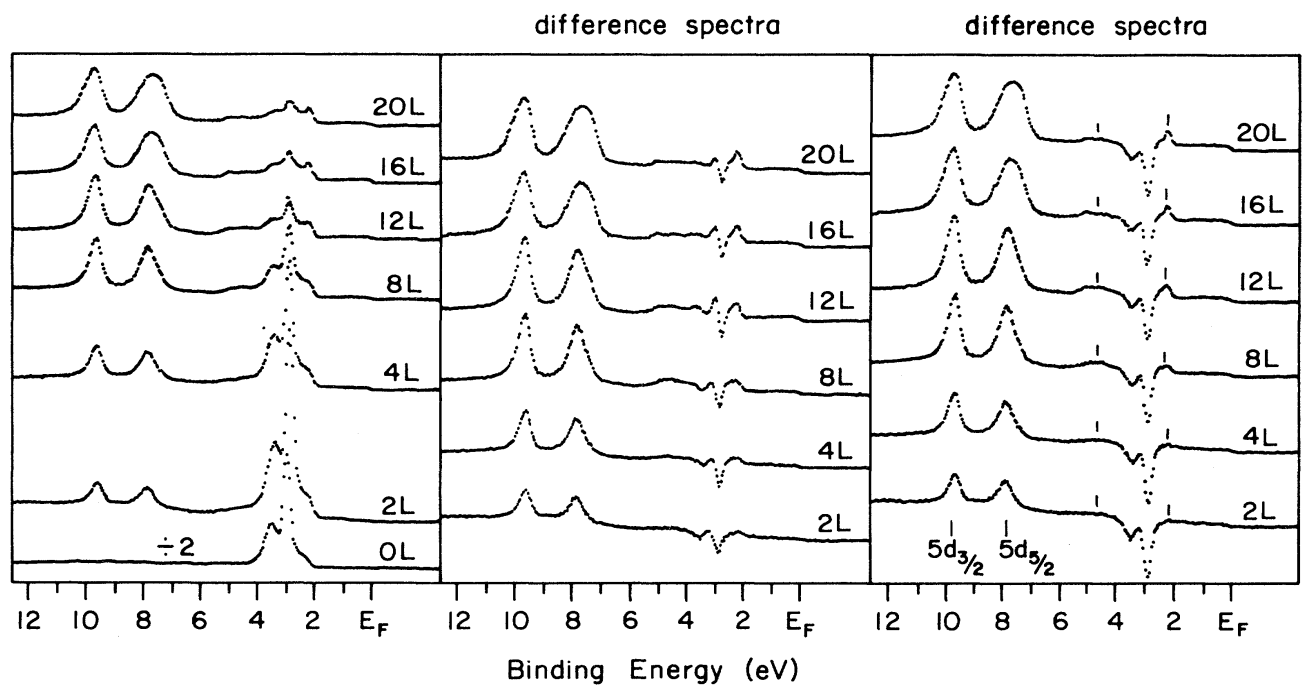

FIG. 7. The photoemission spectra for $\mathrm{Hg}$ adsorption on $\mathrm{Cu}(100)$ at $200 \mathrm{~K}$. All the spectra were taken at a photon energy of $50 \mathrm{eV}$ at normal emission. The light incidence angle is $60^{\circ}$ off the normal. The difference spectra were obtained from the raw spectrum (left) by substracting the $\mathrm{Cu}(100)$ photoemission spectrum, with different weights given to the clean $\mathrm{Cu}(100)$ spectrum (middle and right). $\mathrm{Hg}$-induced features readily apparent in the difference spectra are indicated (right).

$\mathrm{Hg} 5 d$ features now exhibit binding energies of $9.85 \pm 0.1$ and $7.83 \pm 0.1 \mathrm{eV}$ with a full width at half maximum of 0.74 and $1.22 \mathrm{eV}$. The broad $\mathrm{Hg}$-induced feature at $4.7 \pm 0.3 \mathrm{eV}$ increases in binding energy to $5.2 \pm 0.3 \mathrm{eV}$, and the narrow $\mathrm{Hg}$-induced feature at $2.2 \pm 0.1 \mathrm{eV}$ increases substantially in intensity, as does the $\mathrm{Hg}$-induced intensity near the Fermi energy (Fig. 7).

The photoemission results for $\mathrm{Hg}$ coverages less than that of a complete $c(4 \times 4)$ closely resemble those obtained for gaseous $\mathrm{Hg}$. ${ }^{48}$ The photoemission features are narrow and include no third $5 d$ feature, and an $s$-like state can be discerned at about a 5-eV smaller binding energy than the $5 d_{5 / 2}$. The branching ratios for the $\mathrm{Hg} 5 d$ levels are close to the statistical value of 1.5 , and the photon-energy dependence of both the branching ratio and the partial cross sections are quite similar ${ }^{47}$ to those measured for the gaseous $\mathrm{Hg}{ }^{49-52}$

For two monolayers of $\mathrm{Hg}$ on $\mathrm{Cu}(100)$, the increase in the $\mathrm{Hg} 5 d$ level half-widths indicates a change in the chemical environment for the $\mathrm{Hg}$ overlayer. Since the spin-orbit splitting of the $\mathrm{Hg} 5 d$ levels is unlikely to change $^{53}$ from the value near $1.8 \mathrm{eV},{ }^{30}$ the $\mathrm{Hg}$-inducedfeature half-widths imply two overlapping spin-orbit-split pairs of $\mathrm{Hg}$ features, each with a splitting of $1.8 \mathrm{eV}$ (schematically shown in Fig. 8). Comparison of the spectra for the two monolayers of $\mathrm{Hg}$ with the spectra for one monlayer [coverages up to the $c(4 \times 4)$ ] of $\mathrm{Hg}$ permits us to deconvolute the two-monolayer spectra into two $\mathrm{Hg}$ $5 d_{5 / 2}$ features at $9.65 \pm 0.1$ and $10.3 \pm 0.1 \mathrm{eV}$, as described in detail elsewhere. ${ }^{46}$

Upon going from one to two monolayers, the halfwidth of the $\mathrm{Hg} 5 d_{3 / 2}$ feature increases by $27 \%$ and the $\mathrm{Hg} 5 d_{5 / 2}$ feature increases by roughly $50 \%$. We must

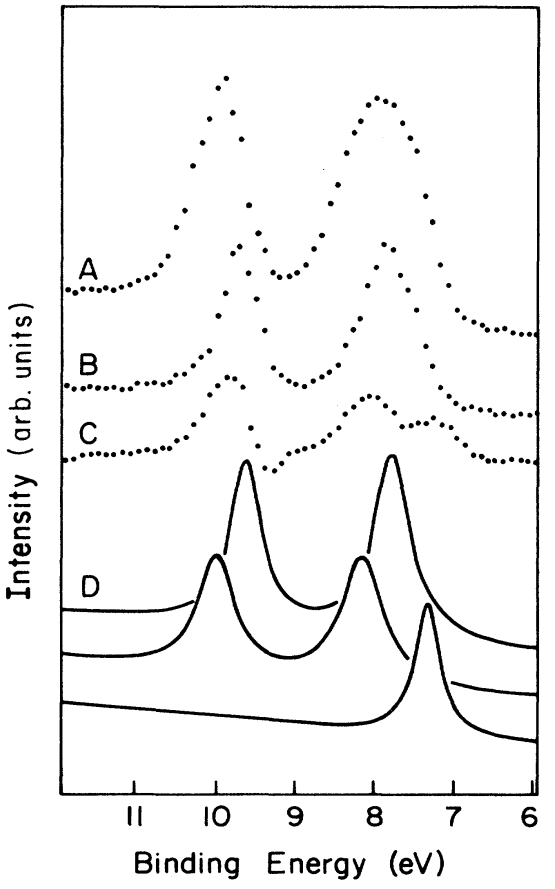

FIG. 8. Photoemission spectra across the $\mathrm{Hg} 5 d$ features for an $\mathrm{Hg}$ bilayer $(A)$, and an $\mathrm{Hg}$ monolayer $(B)$. Note that the difference spectra exhibits two $5 d_{5 / 2}$ features $(C)$. Deconvolution assuming fixed spin-orbit splittings and constant halfwidths are shown of the bilayer $(D)$. The photon energy was 40 $\mathrm{eV}$ and all photoelectrons were collected normal to the surface. 


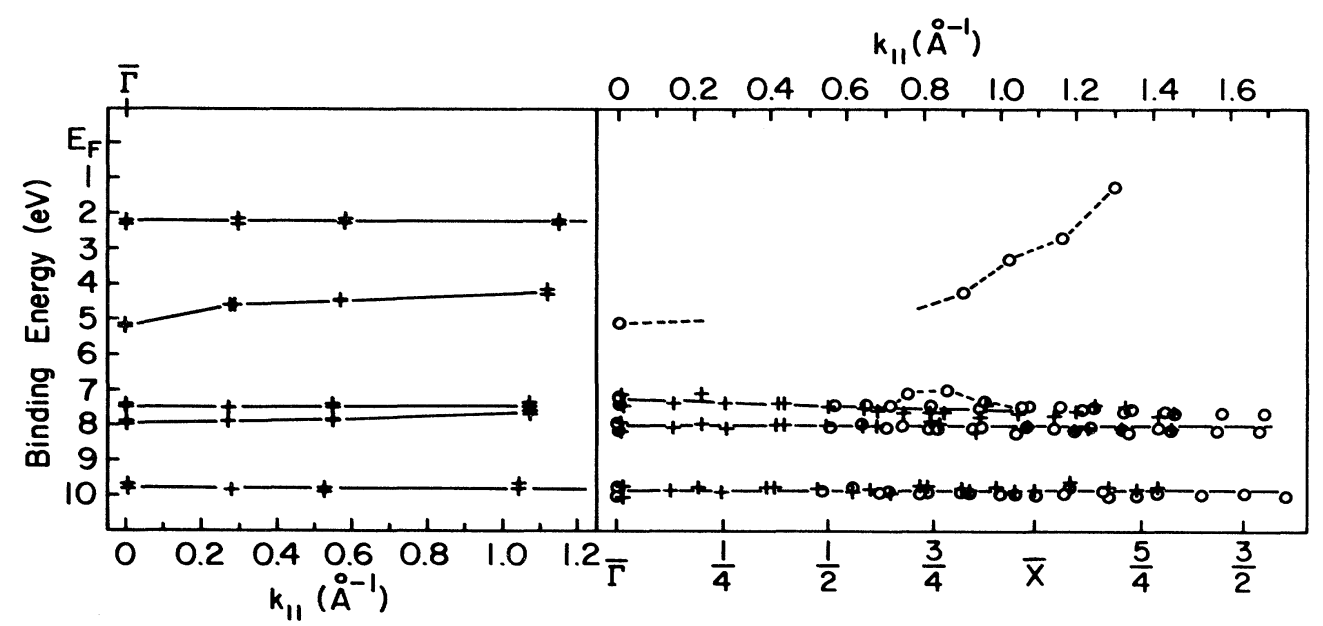

FIG. 9. The experimental band structures of the $\mathrm{Hg}$ bilayer on $\mathrm{Cu}(100)$ (left) and on $\mathrm{Ag}(100)$ (right) neglecting the $\mathrm{Hg}$-induced density of states near the Fermi energy. The band structure for $\mathrm{Hg}$ on $\mathrm{Cu}(100)$ was determined in $p$-polarized light, from photoemission spectra taken at a variety of emission angles. Some states are inferred from difference spectra (see text) and have binding energies that are therefore unreliable. The band structure for $\mathrm{Hg}$ on $\mathrm{Ag}(100)$ was determined for both $s-(+)$ and $p$ - $(0)$ polarized light, and the results are taken from Ref. 43. The dashed lines indicate bands that are only observed in $s$-polarized light. The substrate bands have not been plotted.

therefore conclude that the apparent feature at $7.83 \mathrm{eV}$ observed with two monolayers is an envelope for an $\mathrm{Hg}$ $5 d_{5 / 2}$ feature that contains three features, since both half-widths should otherwise increase by the same amount. By taking the difference between the photoemission spectrum obtained for one monolayer of $\mathrm{Hg}$ and two monolayers of $\mathrm{Hg}$ (Fig. 8), we can also infer that there are, for the latter photoemission spectra, at least five $\mathrm{Hg}$ $5 d$ features for the $\mathrm{Hg}$ bilayers.

Using this analysis, from emission-angle-dependent spectra we find a small dispersion of the $5 d_{5 / 2}$ bands relative to one another with increasing emission angle, as indicated in the experimental band structure plotted in Fig. 9. This result is also consistent with the small decrease in half-width of the $5 d_{5 / 2}$ feature with increasing emission angle (by almost $0.2 \mathrm{eV}$ ). The $\mathrm{Hg} 5 d$ level binding-energy shifts between the underlayer and the topmost layer (or even the monolayer and the bilayer) have been attribut$\mathrm{ed}^{46}$ to a core-level binding-energy shift akin to the surface to bulk core-level shift.

By subtracting the clean $\mathrm{Cu}(100)$ spectra and spectra of $\mathrm{Cu}(100)$ following the adsorption of a small amount of $\mathrm{Hg}(4-6 \mathrm{~L})$, we have attempted to discern if the other $\mathrm{Hg}$-induced features disperse with increasing emission angle. A small decrease in the binding energy from 5.2 to $4.2 \mathrm{eV}$ is observed in increasing the emission angle from normal emission to $20^{\circ}$ for a photon energy of $50 \mathrm{eV}$. Greater dispersions in this feature are difficult to infer because of substantial copper-substrate signal contributions, which limit the value of background subtraction from the photoemission spectra.

Neglecting the $\mathrm{Hg}$-induced density of states near the Fermi energy, from the emission-angle-dependent spectra the band structure of the states induced by adsorption of a bilayer on $\mathrm{Cu}(100)$ has been plotted in Fig. 9. Apart from the narrow $\mathrm{Hg}$-induced feature at about $2.2 \mathrm{eV}$, the band structure of the $\mathrm{Hg}$ bilayer resembles that obtained for the $1 \times 1$ epitaxial $\mathrm{Hg}$ bilayer on $\mathrm{Ag}(100)$ (Ref. 43), as also plotted in Fig. 9. Indeed, theoretical band structures for a free-standing hexagonal monolayer of $\mathrm{Hg}$ (Ref. 5) and liquid $\mathrm{Hg}$ (Ref. 54) also resemble the band structure for $\mathrm{Hg}$ bilayers on $\mathrm{Cu}(100)$ and $\mathrm{Ag}(100)$, apart from emission-angle (wave-vector) -independent features at 2.2-eV binding energy and near the Fermi level, as seen in Fig. 10.

The $\mathrm{Hg}$ bands with a binding energy of $5.2 \mathrm{eV}$ on $\mathrm{Cu}(100)$ and $5.0 \mathrm{eV}$ on $\mathrm{Ag}(100)$ are only observed to disperse to smaller binding energies away from $\bar{\Gamma}$ (normal emission and $\mathbf{k}_{11}=0$ ) for the $\mathrm{Hg}$ bilayer. Dispersion of this band is not observed for the monolayer on either $\mathrm{Ag}(100)$ (Ref. 43) or on $\mathrm{Cu}(100)$. This band is observed for $\operatorname{Ag}(100)$ in even geometry and with $p$-polarized light

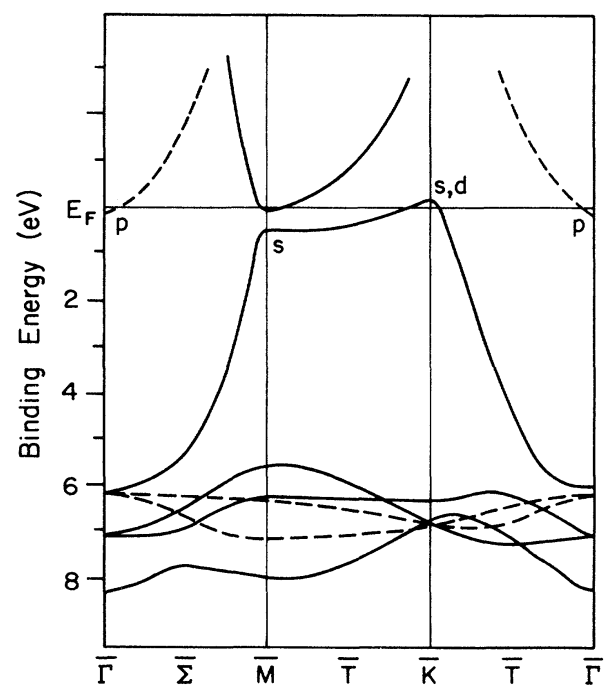

FIG. 10. Theoretical band structure for a free-standing hexagonal monolayer of $\mathrm{Hg}$ taken from Ref. 5. 
but not with $s$-polarized light or in odd geometry. ${ }^{43}$ For $\mathrm{Hg}$ on $\mathrm{Cu}(100)$ this band is most clearly identified with $p$ polarized light. These results strongly indicate that the band is of $\Delta_{1}$ character. The atomic orbitals $s, p_{z}$, and $d_{3 z^{2}-r^{2}}$ orbitals contribute to this representation. With this band character and comparison with the calculated band structures for $\mathrm{Hg},{ }^{5,54}$ we can assign this band as an $s d$-like band.

This $s d$ band does not disperse symmetrically about the surface-Brillouin-zone edge for either the $\mathrm{Hg}$ bilayer on $\mathrm{Cu}(100)$ or $\mathrm{Ag}(100){ }^{43}$ In previous photoemission studies, ${ }^{43}$ the lack of symmetry in the dispersion of this band about the high symmetry of the surface Brillouin zone was explained by strong hybridization with substrate band of similar character and binding energy. Recent studies of Ag overlayers ${ }^{55}$ have shown that quantum-well states resulting from formation of thin metal overlayers on metal substrates can result in the formation of bands with dispersion relationships in $\mathbf{k}_{11}$ that are parabolic or free-electron like [i.e., $E_{n}=\hbar^{2} \mathbf{k}_{11}^{2} /\left(2 m_{11}^{*}\right)$, where $m_{11}^{*}$ is the effective in-plane mass]. Although overlayer hybridization with the substrate must be included, because of the lack of symmetry about the Brillouin-zone edge, the $s d$ band dispersion relationship we have obtained for the $\mathrm{Hg}$ bilayers on $\mathrm{Cu}(100)$ and $\mathrm{Ag}(100)$ states resembles those of quantum-well states. ${ }^{55}$ This band structure is strongly indicative of the metallic character of the mercury bilayer on $\mathrm{Cu}(100)$ at $200 \mathrm{~K}$.

The $\mathrm{Hg}$ density of states at 2.2-eV binding energy and near the Fermi energy do not appear to disperse with variation in $\mathbf{k}_{11}$. These states are difficult to identify, particularly the feature near the Fermi energy. For lattice constants larger than those observed for the metallic phase, Mott ${ }^{56,57}$ proposed that the insulator may have bands that are hybrid bands (such as an $s p$ band), but only through localized states resulting in the Mott pseudogap. Similar localized hybrid states could result in the formation of the bands observed near $E_{F}$ for $\mathrm{Hg}$ on $\mathrm{Ag}(100)$ and $\mathrm{Cu}(100)$ and at $2.2-\mathrm{eV}$ binding energy for $\mathrm{Hg}$ overlayers on $\mathrm{Cu}(100)$, and would explain the absence of any observed dispersion. For gaseous $\mathbf{H g}$, initial-state configuration interactions result in photoemission satellites to the $s$-like orbital. ${ }^{49,58}$ In other words, the ground state of mercury may best be described not by $5 p^{6} 5 d^{10} 6 s^{2}$ $\left({ }^{1} S\right)$, but by $\psi\left({ }^{1} S\right)=a\left(6 s^{2}\right)+b\left(6 p^{2}\right)+\cdots$, where $a$ and $b$ are the configuration mixing coefficients. ${ }^{49}$

By comparing intensity at the Fermi energy for normal emission $\left(\mathbf{k}_{11}=\mathbf{0}\right)$ with the $\mathrm{Hg} 5 d_{3 / 2}$ intensity (which is not as sensitive as the $5 d_{5 / 2}$ level to the formation of band structure ${ }^{30}$ ), a relative measure of the $\mathrm{Hg}$ density of states can be constructed. With use of photoemission spectra obtained for a range of photon energies between 30 and $60 \mathrm{eV}$, a relative density of states has been obtained for $\mathrm{Hg}$ monolayers with a wide variety of lattice constants for square lattices using $\mathrm{Hg}$ adsorbed on $\mathrm{Cu}_{3} \mathrm{Au}(100)(a=2.72 \AA), \operatorname{Ag}(100)(a=2.89 \AA), \mathrm{Hg}$ adsorbed into the $c(2 \times 2)$ on $\mathrm{Cu}(100)(a=3.62 \AA)$, and $\mathrm{Hg}$ adsorbed into the $c(4 \times 4)$ on $\mathrm{Cu}(100)(a=3.22 \AA)$. The results are plotted in Fig. 11, and they compare well, within experimental accuracy, with calculated densities of states ${ }^{5}$ for free-standing monolayers in a square lattice.

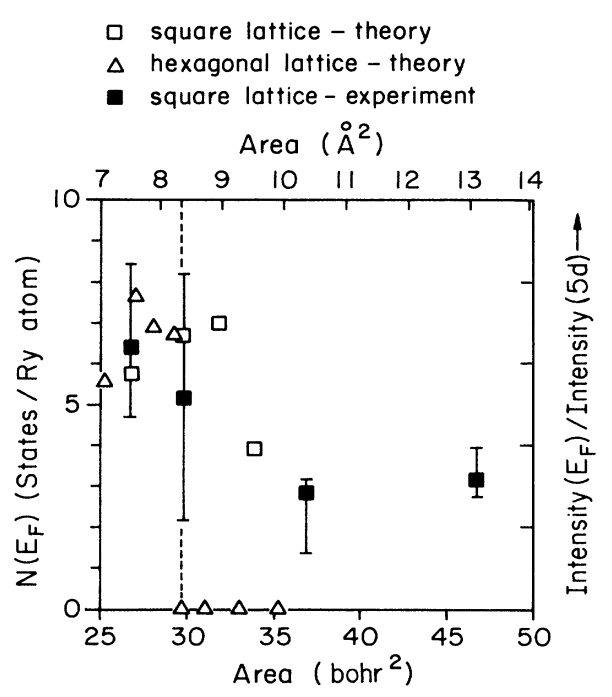

FIG. 11. Calculated densities of states at the Fermi enegy as a function of atomic area for a square lattice $(\square)$ and a hexagonal lattice $(\triangle)$ taken from Ref. 5 . The relative intensity of the $\mathrm{Hg}$-induced photoemission feature at the Fermi energy for a variety of $\mathrm{Hg}$ monolayers in a square lattice are shown as (ש) (see text). The dashed line indicates the predicted metalinsulator transition for a monolayer of $\mathrm{Hg}$ in a hexagonal lattice (Ref. 5) and is in agreement with the structural phase transition observed by Singh and Jones (Refs. 27 and 28).

Singh and Jones ${ }^{27,28}$ have observed structural transitions for monolayer films of $\mathrm{Hg}$ on $\mathrm{Ni}(111)$ that appear to be driven by the density of states near the Fermi energy, which is also consistent with the calculated density of states shown in Fig. 11. It is very clear for low photon energies (about $30 \mathrm{eV}$ ) or high photon energies (about 80 $\mathrm{eV}$ ) that there is an increase in the apparent density of states (at normal emission) at the Fermi energy upon formation of the mercury bilayer (as compared with the mercury monolayer). Such a change can be indicative of more metallic character for the mercury bilayer than is the case for the mercury monolayer. Indentifying whether these states near $E_{F}$ are localized or itinerant becomes important.

The absence of $\mathrm{Cu}(100)$ intensity near the Fermi level for photon energies between 40 and $70 \mathrm{eV}$ and the apparent $\mathrm{Hg}$-induced intensity near $E_{F}$ with increasing $\mathrm{Hg}$ coverages on $\mathrm{Cu}(100)$ provide us with a means for probing the $\mathrm{Hg}$ partial photoemission cross section not only for the $\mathrm{Hg} 5 d$ levels, but also for states near the Fermi energy, so long as such constant-initial-state spectra are taken at $\mathbf{k}_{11}=0$, i.e., normal emission.

\section{THE PARTIAL PHOTOEMISSION CROSS SECTIONS OF Hg VALENCE LEVELS FOR Hg OVERLAYERS ON Cu(100)}

By taking constant-initial-state spectra with the resolution of both the monochromator and the electron-energy analyzer degraded, an estimate of the various partial photoemission partial cross sections may be obtained. At 


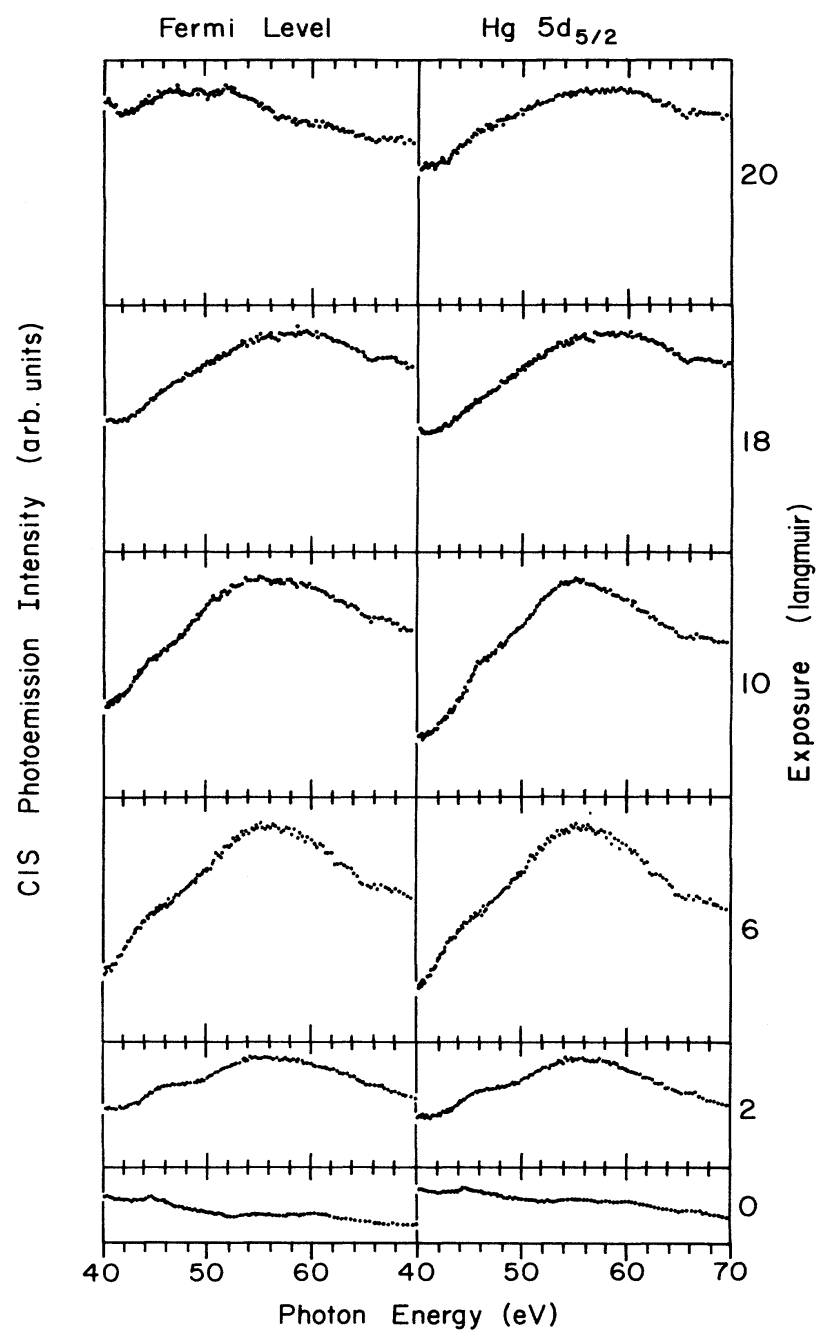

FIg. 12. Constant initial-state spectra for $\mathrm{Hg}$ adsorbed on $\mathrm{Cu}(100)$ at $200 \mathrm{~K}$. The spectra are taken for the $\mathrm{Hg}$ initial states of $5 d_{5 / 2}$ and just below the Fermi energy. For each initial state, the spectra are plotted on the same relative scale. The light incidence angle is $60^{\circ}$ off the surface normal, and the photoelectrons are collected normal to the surface. Note the decrease in signal variation for $\mathrm{Hg}$ exposures greater than $10 \mathrm{~L}$.

normal emission, we have taken such CIS spectra for both the occupied states near the Fermi energy and for the $\mathrm{Hg} 5 d_{5 / 2}$ levels for a variety of $\mathrm{Hg}$ coverages, as seen in Fig. 12.

The $\mathrm{Cu}(100)$ substrate is seen to contribute little photoemission intensity for photon energies between 40 and 70 $\mathrm{eV}$ (as seen in Fig. 12), consistent with the photoemission energy-distribution curves (Fig. 7). With the adsorption of $\mathrm{Hg}$ on $\mathrm{Cu}(100)$ at $200 \mathrm{~K}$, a broad feature becomes apparent in the CIS spectra for both the states near the Fermi level and the $5 d_{5 / 2}$ levels, as has been reported elsewhere. ${ }^{47,59-60}$ This broad feature, which is centered about a photon energy of 55-56 eV, increases with increasing $\mathrm{Hg}$ exposure (Fig. 12) until completion of the $c(4 \times 4)$ at about $10-12 \mathrm{~L}$. As has been noticed in prior studies, ${ }^{47}$ the CIS spectra do show that this broad feature in the CIS spectra becomes broader as the lattice constant decreases [from $3.62 \AA$ for the $c(2 \times 2)$ to $3.22 \AA$ for the $c(4 \times 4)]$, as seen for CIS spectra taken for a very wide photon-energy range from the $\mathrm{Hg} 5 d_{5 / 2}$ levels (Fig. 13). With adsorption of the second layer, the $\mathrm{Hg}$-induced features continue to increase in intensity, but the variation in intensity with photon energy declines (Fig. 12). With completion of the second layer, the variation in intensity as determined from CIS spectra, across the photon-energy range $40-70 \mathrm{eV}$, is negligible, despite the substantially greater amount of $\mathrm{Hg}$ in the bilayers than is observed in the $\mathbf{H g}$ monolayer.

The broad feature in these partial photoemission cross sections is due to the $5 d \rightarrow \epsilon f$ excitation, with the $5 d \rightarrow \epsilon p$ cross-section contribution decreasing from the threshold. ${ }^{47,60-62}$ These photoemission partial cross sections are very different from those observed for gaseous mercury with the $5 d \rightarrow \epsilon f$ cross-section maximum at a higher energy (Fig. 13) than is the case for gaseous mercury, where the peak occurs at less than $40 \mathrm{eV} .{ }^{52,61,62}$ Both disorder $^{47,60}$ and large lattice constants ${ }^{39,47}$ result in partial $\mathrm{Hg} 5 d$ cross sections, as determined by CIS, that more closely resemble the partial cross sections observed for gaseous mercury. Upon formation of the $\mathrm{Hg}$ bilayer on $\mathrm{Cu}(100)$, not only does the cross section for the $5 d-\epsilon f$ excitation become broader, but the peak in the cross section continues to shift to larger photon energies, as seen in Fig. 13. This shift of $5 \mathrm{eV}$ is most apparent for the $5 d$ levels (Fig. 12).

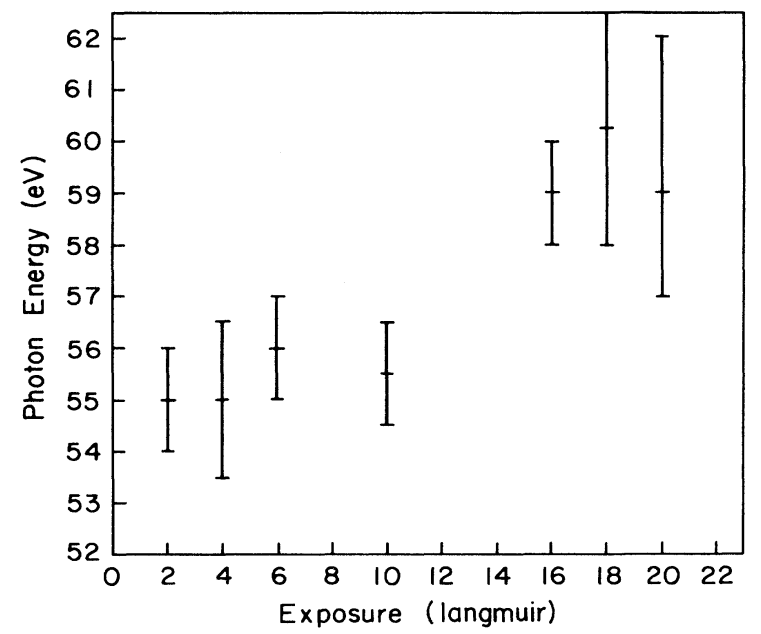

FIG. 13. The photon energy of the peak in the $\mathrm{Hg}$ partial cross sections for various coverages on $\mathrm{Hg}$ on $\mathrm{Cu}(100)$ at $200 \mathrm{~K}$. The partial photoemission cross-section peak energies are determined from CIS spectra taken off both the $\mathrm{Hg} 5 d$ levels and the $\mathrm{Hg}$ states near the Fermi energy for CIS taken in normal emission. 


\section{BARIUM OVERLAYERS ON Ni(111)}

The photoemission spectra for 3,8 , and $30 \AA$ of barium deposited on $\mathrm{Ni}(111)$ are illustrated in Fig. 14. The large density of states is largely attributable to the nickel $3 d$ states, and this density of states obscures the relatively weak $\mathrm{Ba} 6 s p$ band. ${ }^{63}$ This problem is also apparent in studies of $\mathrm{Hg}$ adsorption on $\mathrm{Ni}(111) .{ }^{27-29}$ The features at approximately $14.7-$ and $16.5-\mathrm{eV}$ binding energies are, respectively, the $\mathrm{Ba} 5 p_{3 / 2}$ and $5 p_{1 / 2}$ spin-orbit-split doublet, and have been observed by Richter et $a l .{ }^{33}$ for the free barium atom and by $\mathrm{Hecht}^{34}$ for barium thin films. The spin-orbit splitting of the barium $5 p$ doublet is $1.9 \pm 0.1 \mathrm{eV}$, in good agreement with the value reported by Ley, Marlensson, and Azouly ${ }^{64}$ of $2.0 \mathrm{eV}$. Unlike Ca and $\mathrm{Sr}$, the $5 p$ shallow-core-level spin-orbit splitting changes little between the free atom and the condensed metal. ${ }^{64}$ It is therefore unsurprising that we also observe little coverage-dependent change in the spin-orbit splitting within the accuracy of our experiment.

The $5 p$ binding energies do depend upon bariumoverlayer thickness, as indicated in Figs. 14 and 15. At very low coverages the barium $5 p_{3 / 2}$ and $5 p_{1 / 2}$ binding energies are $14.8 \pm 0.1$ and $16.8 \pm 0.1 \mathrm{eV}$, respectively. At higher coverages (at a nominal thichness of $6 \AA$ ), the binding energies decrease to $14.3 \pm 0.1$ and $16.1 \pm 0.1 \mathrm{eV}$, but then slowly increase with further increases in the barium-overlayer coverage to binding energies of $14.9 \pm 0.1$ and $17.2 \pm 0.3 \mathrm{eV}$ at a $30-\AA$ barium-overlayer thickness.

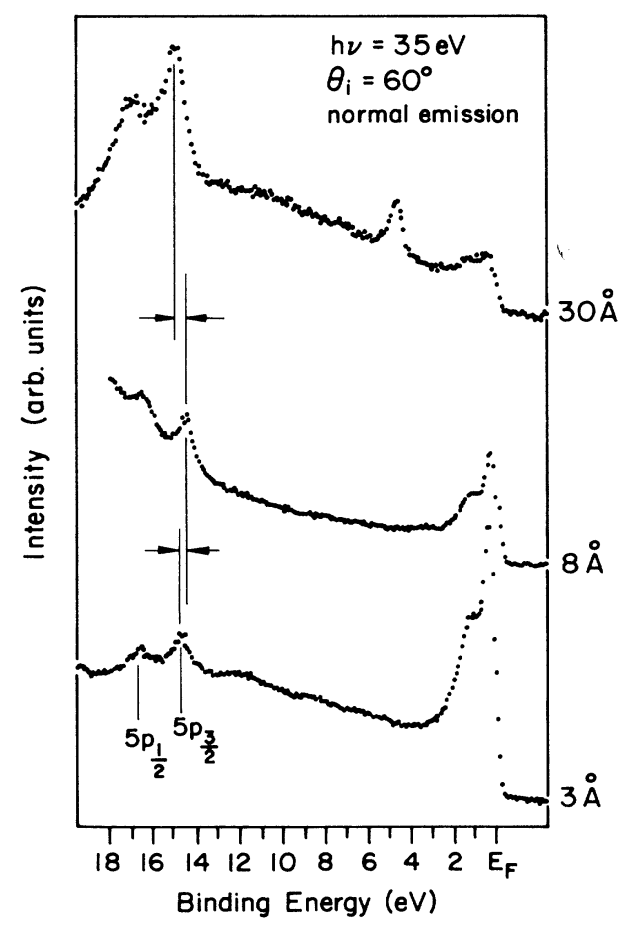

FIG. 14. Photoemission spectra for barium overlayers deposited on $\mathrm{Ni}(111)$. The photon energy for all the spectra is $35 \mathrm{eV}$ with a light incidence angle of $60^{\circ}$ off the surface normal. All photoelectrons were collected normal to the surface.

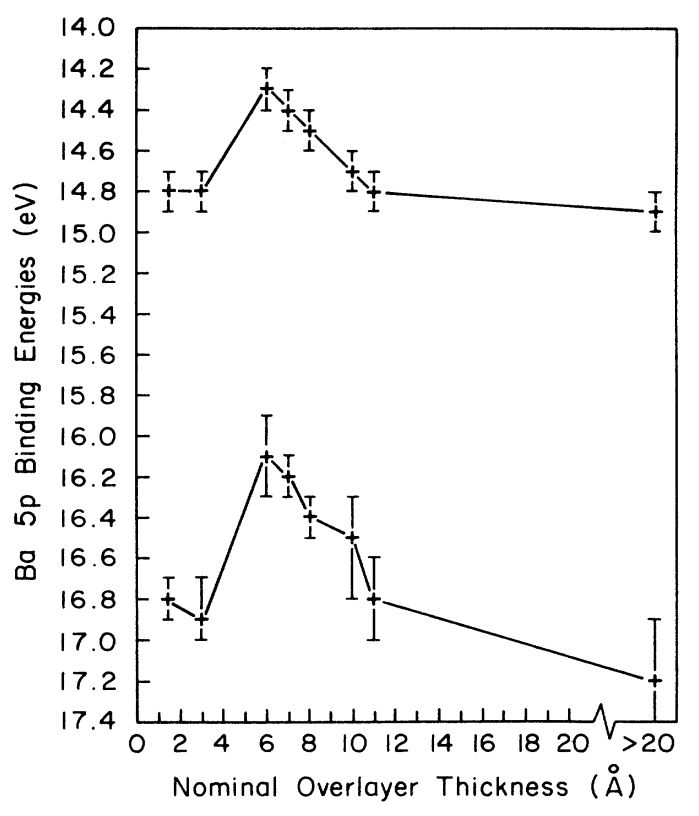

FIG. 15. The barium $5 p$ spin-orbit split binding energies as a function of the barium overlayer thickness. The coverages are only the nominal coverages; the relative coverages are in error by no more than $10 \%$. The binding energies are with respect to the Fermi level determined for the clean Ni(111) substrate.

The barium $5 p$ binding-energy shift of $0.8 \pm 0.2 \mathrm{eV}$ (Ref. 25) with increasing coverage from 6 to $30 \AA$ is qualitatively very similar to the increase in the $\mathrm{Hg} 5 d$ binding energies reported in the previous section and $\mathrm{Au}$ overlayers. ${ }^{65}$ For both $\mathrm{Ba}$ and $\mathrm{Hg}$, we attribute this shallowcore-level binding-energy shift to a core-level shift akin to a surface to bulk core-level shift. ${ }^{66,67}$ The binding-energy decrease of the barium $5 p$ levels with increasing barium coverage from a submonolayer coverage (nominal thickness of $1-3 \AA$ ) to modest coverages $(6-\AA$ nominal thickness) can be explained through a change in the work function upon barium deposition and the resulting charge transfer from the surface barium atoms to the substrate nickel atoms. A change in the surface work function will, of course, only be apparent as a core-level shift in the surface atom and not the bulk. ${ }^{66}$ We have no evidence from our data suggesting the alloying of these very thin films occurs, but alloying at the interface cannot be excluded and may also affect the $5 p$ binding energies.

In order to probe the electronic structure in the vicinity of the Fermi energy for barium overlayers, constantinitial-state spectra were taken in a similar fashion as was done for the $\mathrm{Hg}$ overlayer on $\mathrm{Cu}(100)$. Figure 16 illustrates the CIS spectra for the density of states just below the Fermi energy and for the Ba $5 p_{3 / 2}$ level for bariumoverlayer thicknesses of 3,8 , and $30 \AA$. There are two photoemission resonances of importance to this work on the metal-insulator transition, that can be identified from the CIS spectra. There is a resonance at about $28 \mathrm{eV}$ with an onset at a photon energy just below $25 \mathrm{eV}$ and another resonance at $42 \mathrm{eV}$ with a shape onset at a pho- 
ton energy of about $31 \mathrm{eV}$.

The first resonance at $28 \mathrm{eV}$ has been observed in the ultraviolet absorption spectra of barium ${ }^{68}$ and has been attributed to a $5 p-4 f$ transition, though this transition is dipole-selection-rule forbidden. The $4 f$ levels, when placed just above the Fermi energy, were postulated to increase in binding energy to the binding energy of the occupied $6 s$ level in the final state. The argument proposed is that the far more localized $4 f$ electrons are susceptible to a greater attractive force than the $6 \mathrm{~s}$ electrons to the $\mathrm{Ba} 5 p$ hole. This excitation could result in a photoemission resonance of the $\mathrm{Ba} 6 s(\mathrm{Ni} 3 d)$ level as a result of an intra-atomic electron transition and Auger decay (see Ref. 26 and the references therein):

$$
5 p^{6} 6 s^{2} \rightarrow 5 p^{5} 6 s^{1} 4 f^{1} \rightarrow 5 p^{6} 6 s^{1}+e^{-},
$$

which is identical to the direct-photoemission final state $5 p^{6} 6 s^{1}$. The onset of this resonance is approximately the binding energy of the $5 p$ levels (approximately $16.8 \mathrm{eV}$ for the $5 p_{1 / 2}$ level) plus the energy of the $4 f$ level above the

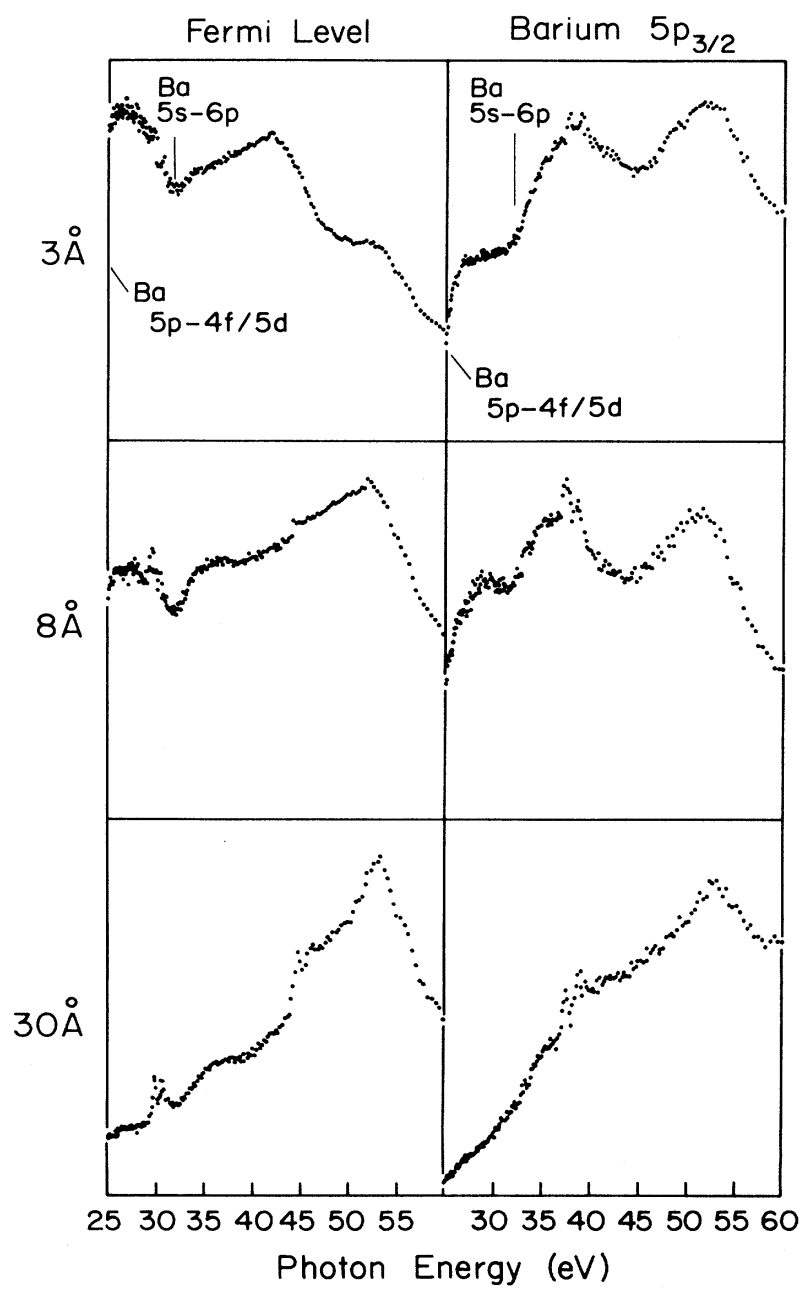

FIG. 16. The constant initial state spectra for 3-, 8-, and 30A-thick barrium overlayers. For each barrium coverage, CIS spectra were taken for the state $0.3 \mathrm{eV}$ below the Fermi level and the barium $5 p_{3 / 2}$ shallow core level.
Fermi energy (determined to be about $9.8 \mathrm{eV}$ above the Fermi energy ${ }^{69-71}$ ), or about $26.6 \mathrm{eV}$. This is in good agreement with our results, and the observed onset energy of the resonance as well as the high density of states of the $4 f$ levels $^{71}$ supports this mechanism. Because this resonance is very strong for the thinner barium overlayers, a photoemission resonance that is not dipoleselection-rule forbidden must also be considered.

Atomic barium, ${ }^{72}$ as is the case for $\mathrm{Hg}$, is a mixture of several initial-state configurations. This, combined with the hybridization of the $6 s$ and $6 p$ levels that must occur in the condensed state (resulting in metallic barium), permits a $5 p$-to- $6 s$ excitation, since barium is no longer strictly $5 p^{6} 6 s^{2}$, but rather $5 p^{6} 6 s^{2-d} 6 p^{d}$ (with $d$ nonzero) in the initial state. This initial state permits a dipoleallowed $5 p$-to- $6 s$ excitation to occur, which is then followed by an Auger-like decay process to fill the $5 p$ hole and will result in a final state identical with the directphotoemission process from the $6 s p$ band. This would have an onset much closer to the $5 p$ binding energy $(16.8$ eV) than the observed threshold near $25 \mathrm{eV}$. Extraatomic excitation to the nickel $3 d$ states could also occur at about the same threshold.

A $5 p$-to- $5 d$ excitation is also possible. ${ }^{72-76}$ A high intensity of Auger peaks was observed at 28.9-eV photon energy for atomic barium ${ }^{76}$ suggesting that such resonance with thresholds of $20-25 \mathrm{eV}$ could be substantial contributors to this resonance ${ }^{72}$ with the excitations

$$
5 p^{6} 6 s^{2} 5 d^{0} \rightarrow 5 p^{5} 6 s^{2} 5 d^{1}
$$

(at a ${ }^{2} P_{3 / 2}$ series limit of $22.7 \mathrm{eV}$ and a ${ }^{2} P_{1 / 2}$ series limit of $24.8 \mathrm{eV}$ ) or

$$
5 p^{6} 6 s p^{2} 5 d^{0} \rightarrow 5 p^{5} 6 p^{2} 5 d^{1},
$$

both possible [because of configuration mixing some $6 \%$ of the initial state of atomic barium is $5 p^{6} 6 p^{2}$ (Ref. 72)], followed by an Auger decay, resulting in a final state identical to the direct photoemission process. This excitation is a super-Coster-Kronig transition and should lead to an intense photoemission resonance. The $\mathrm{Ba} 5 d$ state would be localized in much the same way as proposed for the $5 p$-to- $4 f$ excitation.

The resonance at $42 \mathrm{eV}$ has an onset of $31 \mathrm{eV}$ (Fig. 16), and this is very close to the binding energy of the barium $5 s$ core level at $30.1 \mathrm{eV}$ below the Fermi energy. ${ }^{34,77} \mathrm{~A}$ dipole-allowed transition of the type

$$
5 s^{2} 5 p^{6} 6 s^{2} \rightarrow 5 s^{1} 5 p^{6} 6 s^{2} 6 p^{1},
$$

followed by decay and ejection of an Auger electron,

$$
5 s^{1} 5 p^{6} 6 s^{2} 6 p^{1} \rightarrow 5 s^{2} 5 p^{6} 6 s^{1}+e^{-},
$$

leads to a final state identical to direct emission from the $6 s p$ band. Similarly, a decay filling the $5 s$ hole followed by emission from the $5 p$ core level,

$$
5 s^{1} 5 p^{6} 6 s^{2} 6 p^{1} \rightarrow 5 s^{2} 5 p^{5} s^{2}+e^{-}
$$

results in a final state identical to direct emission from the $\mathrm{Ba} 5 p$ core level. These transitions will lead to photoemission resonance of the $6 s$ and $5 p$ states, respectively. Similar photoemission resonances have been observed as 
a result of $s$-to-p excitations in nickel ${ }^{78}$ and gadolinium. ${ }^{26}$ In general, for such resonances resulting in an $s$-to- $p$ transition, the $p$ state becomes localized as a result of the attraction of the $s$ hole. ${ }^{78}$ The intensity of the photoemission resonance will depend upon the extent of localization of the $p$-like state.

The intensity of both the 28- and 42-eV photoemission resonances for the density of states near the Fermi energy and for the $\mathrm{Ba} 5 p_{3 / 2}$ core level depend on the barium coverage on $\mathrm{Ni}(111)$, as seen in Fig. 16. The resonances are strongest for the thinnest barium films and weak or nonexistent with the very thick barium films. The behavior of these photoemission resonances is similar to the decline in variation with photon energy for the $5 d$-to- $\epsilon f$ excitation cross sections in the photoemission from the $\mathrm{Hg}$ $5 d_{5 / 2}$ state and the states near the Fermi energy for $\mathbf{H g}$ on $\mathrm{Cu}(100)$ (Fig. 12).

\section{DISCUSSION}

Given that both mercury and barium gain metallic character through the hybridization of the closed atomic orbitals between adjacent atoms to form partly filled bands at the Fermi energy, the itinerancy of the states at the Fermi level will depend upon the orbital overlap and the coordination between adjacent mercury or barium atoms. A change in the density of conduction or itinerant electrons will affect a photoemission resonant intensity. ${ }^{19-22}$ The more localized the core-excited electron, the greater is the resonant intensity.

For barium on $\mathrm{Ni}(111)$, we observe a substantial decline in the photoemission resonant intensities at 28 and $42 \mathrm{eV}$ with increasing coverage. Despite the increase in barium coverage, the resonant intensities become negligible upon formation of a barium bilayer (Fig. 16). We can infer that the core excited electron becomes delocalized, or the lifetime of the core exciton formed in the photoemission process become very short. Such behavior is strongly reminiscent of the exciton unbinding that occurs at the Mott metal-nonmetal transition.

For mercury on $\mathrm{Cu}(100)$ at $200 \mathrm{~K}$, the variation in the partial photoemission cross sections for the $5 d$-to- $\epsilon f$ excitation decreases dramatically upon formation of the bilayer (Fig. 12). This can be attributed to the increased screening of the photohole in the photoemission process. ${ }^{60}$ Such increased screening is strongly indicative of greater orbital hybridization or greater itinerancy of the valence-electron bands. Existing calculations, ${ }^{79}$ although only considering nearest-neighbor interactions, have indicated that such a shift of the $5 d$-to- $\epsilon f$ excitation to higher photon energies (as seen in Fig. 13) is consistent with a change in the exchange-correlation potential for the interaction between a given atomic orbital and the surrounding atoms from a localized one-electron, selfconsistent-field potential to a potential accommodating free-electron states. From the band structures determined for $\mathrm{Hg}$ bilayers, it is clear that the bilayer does possess some very itinerant (and in some instances, nearly-free-electron-like) bands (Fig. 9) supporting the postulate that the bilayer is more metallic in character.

For both barium on $\mathrm{Ni}(111)$ and mercury on $\mathrm{Cu}(100)$, upon formation of the bilayer, the bands become much less localized and the film becomes increasingly metallic in behavior, in the photoemission final state. This transition to metallic behavior upon formation of the bilayer has also been observed for $\mathrm{Cs}$ on GaAs surface. ${ }^{80}$ Though the substrate for the Cs overlayers possessed a band gap, the behavior does resemble that observed with barium and mercury overlayers.

Experimental $^{81-83}$ and theoretical ${ }^{6,84}$ studies of mercury clusters have shown that there is a gradual transition to metallic character with increasing cluster size. An average coordination number of 5 to 7 per mercury atom is needed to ensure some metallic character, with fully metallic character apparent with a coordination number of 10 to 11 . From our structural studies, we know that the second layer of mercury atoms initially adsorbs into the high-coordination sites. Thus, for mercury on $\mathrm{Cu}(100)$ at $200 \mathrm{~K}$, growth of the second layer results in a dramatic increase in the average coordination number per overlayer atom. The increasing metallic character observed with formation of the mercury bilayer is, therefore, consistent with the results obtained for mercury clusters. By adsorbing mercury on $\mathrm{Ag}(100)$ at $30 \mathrm{~K}$ (Ref. 60 ) it was observed that the partial cross sections closely resemble those of gaseous mercury. Such a result suggests that electronic states in films with a very large number of defects are far more localized than is the case for well-ordered films. If we regard defects as reducing the "average cluster size" of ordered aggregates of mercury, then this also suggests that the behavior of thin films reflects the metal-insulator transition results observed for mercury clusters.

The lattice constant of the $c(2 \times 2)(3.62 \AA)$ and the $c(4 \times 4)(3.22 \AA)$ for submonolayer films of $\mathrm{Hg}$ on $\mathrm{Cu}(100)$ at $200 \mathrm{~K}$ are smaller than the critical lattice constants for fcc and bcc mercury. ${ }^{85}$ The critical lattice constants for the metal-insulator transition in expanded mercury are about 5.1-5.9 $\AA$ for fcc mercury and 4.2-5.0 $\AA$ for bcc mercury. If these critical lattice constants are reasonably reliable, the observation that the electronic structure, particularly for states near the Fermi energy, is representative of localized states in the photoemission final state for monolayer films of $\mathrm{Hg}$, suggests that both coordination number of the atoms in the thin films, as well as lattice constant, are crucial for metallic behavior. As films grow, the coordination number of atoms at the surface is lower than will be the case for atoms within the bulk of the film. This, together with the presence of surface defects, may result in insulating or nonmetallic surface layers for divalent metal crystals, consistent with theoretical suggestions. ${ }^{6}$

The mixed configurations that are possible for divalent metals such as barium and mercury result in densities of states near the Fermi energy. Unfortunately, these densities of states (despite theoretical models ${ }^{5}$ that agree with our results) are not necessarily a reliable indicator of metallic behavior for mercury, as indicated in Fig. 11. At least for the excited state, resonant photoemission does seem to be a reliable and useful indicator of metallic behavior.

That a $c(4 \times 4) \mathrm{Hg}$ overlayer on $\mathrm{Cu}(100)$ appears to be 
akin to a nonmetallic (insulating in fact) layer is unsurprising, in view of other $\mathrm{Hg}$-overlayer studies. The lattice constant for this overlayer is $3.22 \AA$, and is larger than that for the square $\mathrm{Hg}$ lattice of $\mathrm{Hg}$ adsorbed on $\mathrm{W}(100)$ of $3.16 \AA{ }^{86}$ Apart from a $2.6-\mathrm{eV}$ shift of the ${ }^{2} S_{1 / 2},{ }^{2} D_{5 / 2}$, and ${ }^{2} D_{3 / 2}$ peaks [at binding energies of 3.2 , 7.6, and $9.6 \mathrm{eV}$ below the Fermi energy for $\mathrm{Hg}$ on $\mathrm{W}(100)$ ] relative to the gas phase due to screening and polarization effects of the metal substrate, ${ }^{87}$ the $\mathrm{Hg}$ overlayer on W(100) exhibits an electronic structure very similar to the gas phase and may be thought of as "nonmetallic". ${ }^{87}$

\section{CONCLUSION}

The valence-electronic states for barium monolayers on $\mathrm{Ni}(111)$ and for mercury monolayers on $\mathrm{Cu}(100)$ at $200 \mathrm{~K}$ appear to be highly localized and become highly metallic in nature upon formation of the bilayer. The $\mathrm{Hg}$-band structure and CIS spectra for both the barium and the mercury thin films indicate that there is some transition in the electronic structure upon formation of the bilayer toward a more metallic thin film. The core excition formed in the resonant photoemission process unbinds in much the same manner as predicted for the Mott-type metal-nonmetal insulator transition. The behavior of these divalent-metal thin films in the photoemission final state resembles the initial-state semiconductor-metal transition observed with cesium thin films. Clearly, resonant photoemission can be used to probe changes in the itinerancy of electrons in the valence bands, even when photoemission signals from the substrate are substantial.

We postulate, on the basis of this work and previous work with divalent-metal clusters, ${ }^{81-84}$ that both the lattice constant and the coordination number are important for ensuring metallic character. These results indicate that divalent-metal thin films are rich in metallic and nonmetallic phases associated with different structural phases, as suggested by theory. ${ }^{5}$

\section{ACKNOWLEDGMENTS}

The authors would like to thank Dr. Klaus Rademann (Philipps Universität Marburg), Dr. Walter Lambrecht (Case Western Reserve University), and Jiandi Zhang (Syracuse University) for pointing out some additional facets to the metal-insulator transition, and Y. J. Kime, Wei $\mathbf{L i}$, and Charles Hutchings for their considerable technical assistance. This work was funded by the NSF through Grant No. DMR 88-20779. The authors would also like to thank the staff of the Synchrotron Radiation Center (SRC) in Stoughton, Wisconsin where this work was carried out. The SRC is funded by the NSF.
${ }^{*}$ To whom correspondence may be addressed.

${ }^{1}$ F. Yonezawa and T. Ogawa, Prog. Theor. Phys. Suppl. 72, 1 (1982), and references therein.

${ }^{2}$ Tohru Ogawa, S. Nara, and T. Matsubara, Prog. Theor. Phys. Suppl. 72, 140 (1982).

${ }^{3}$ L. A. Turkevich and M. H. Cohen, Phys. Rev. Lett. 53, 2323 (1984).

${ }^{4}$ N. E. Cusack, Prog. Theor. Phys., Suppl. 72, 81 (1982), and references therein.

${ }^{5}$ H. J. F. Jansen, A. J. Freeman, M. Weinert, and E. Wimmer, Phys. Rev. B 28, 593 (1983).

${ }^{6}$ A. R. Miedema and Dorleijn, Philos. Mag. B 43, 251 (1981).

${ }^{7}$ A. Yoneda and S. Endo, J. Appl. Phys. 51, 3216 (1980); A. S. Balchan and H. G. Drickamer, Rev. Sci. Instrum. 32, 308 (1961).

${ }^{8}$ U. El-Hanany, and W. W. Warren, Jr., Phys. Rev. Lett. 34, $1276(1975)$.

${ }^{9}$ W. W. Warren, Jr. and F. Hensel, Phys. Rev. B 26, 5980 (1982).

${ }^{10}$ U. Even and J. Jortner, Phys. Rev. Lett. 28, 31 (1972).

${ }^{11}$ E. U. Frank and F. Hensel, Phys. Rev. 147, 109 (1966).

${ }^{12} \mathrm{O}$. Cheshnovksi, U. Even, and J. Jortner, Solid State Commun. 22, 745 (1977).

${ }^{13}$ H. Uchtmann and F. Hensel, Phys. Lett. 53A, 239 (1975).

${ }^{14}$ W. Hefner and F. Hensel, Phys. Rev. Lett. 48, 1026 (1982).

${ }^{15}$ H. K. Schwarzenegger, A. L. Simmons, A. L. Passner, and S. L. McCall, Phys. Rev. B 18, 2494 (1978).

${ }^{16}$ C. E. Krohn and J. C. Thompson, Phys. Rev. B 21, 2619 (1980).

${ }^{17}$ N. F. Mott, Rev. Mod. Phys. 40, 677 (1968).

${ }^{18}$ N. F. Mott, Metal Insulator Transitions, 2nd ed. (Taylor and Francis, London, 1990).

${ }^{19}$ S. Hufner and G. K. Wertheim, Phys. Lett. 51A, 299 (1975).
${ }^{20}$ J. Friedel, Philos. Mag. 43, 153 (1952).

${ }^{21}$ J. Friedel, Adv. Phys. 3, 446 (1954).

${ }^{22}$ M. Combescot and P. Nozières, J. Phys. (Paris) 32, 913 (1971).

${ }^{23}$ A. Kotani and Y. Toyozawa, J. Phys. Soc. Jpn. 37, 912 (1974).

${ }^{24}$ A. Kotani, Jpn. J. Appl. Phys. 17, Suppl. 17-2, 243 (1978).

${ }^{25}$ P. A. Dowben and D. LaGraffe, Phys. Lett. A 144, 193, (1990).

${ }^{26}$ P. A. Dowben, D. LaGraffe, and M. Onellion, J. Phys. Condens. Matter 1, 6571 (1989).

${ }^{27}$ N. K. Singh and R. G. Jones, Chem. Phys. Lett. 155, 463 (1988).

${ }^{28}$ N. K. Singh and R. G. Jones, Surf. Sci. 232, 243 (1990).

${ }^{29}$ N. K. Singh and R. G. Jones, J. Phys. Condens. Matter 1, SB267 (1989).

${ }^{30}$ P. A. Dowben, S. Varma, Y. J. Kime, D. R. Mueller, and M. Onellion, Z. Phys. B 73, 247 (1988); M. Onellion, Y. J. Kime, P. A. Dowben, and N. Tache, J. Phys. D 20, L633 (1987), and references therein.

${ }^{31}$ M. Ya. Amusia, V. K. Ivanov, and L. V. Chernysheva, Phys. Lett. 59A, 191 (1976).

${ }^{32}$ A. Zangwill and P. Soven, Phys. Rev. Lett. 45, 204 (1980).

${ }^{33}$ H. P. Richter, M. Meyer, P. Pahler, T. Prescher, E. von Raven, B. Sonntag, and H. E. Wetzel, Phys. Rev. A 39, 5666 (1989).

${ }^{34}$ M. H. Hecht, Ph.D. dissertation, Stanford University, 1982 (unpublished).

${ }^{35}$ H. P. Kelly, S. L. Carter, and B. E. Norum, Phys. Rev. A 25, 2052 (1982).

${ }^{36}$ A. Shih, C. Hor, D. Mueller, C. R. K. Marrian, W. T. Elam, P. Wolf, J. P. Kirkland, and R. A. Neiser, J. Vac. Sci. Technol. A 6, 1058 (1988).

${ }^{37}$ J. A. T. Verhoeven and H. van Doveren, Appl. Surf. Sci. 5, 
361 (1980); B. C. Lamartine, J. v. Czarnecki, and T. W. Haas, ibid. 16, 207 (1983).

${ }^{38}$ R. E. Thomas, A. Shih, and G. A. Haas, Surf. Sci. 75, 239 (1978).

${ }^{39}$ P. A. Dowben, Y. J. Kime, C. W. Hutchings, Wei Li, and G. Vidali, Surf. Sci. 230, 113 (1990); C. W. Hutchings, P. A. Dowben, Y. J. Kime, W. Li, M. Karimi, C. Moses, and G. Vidali, in Atomic Scale Structure of Interfaces, edited by R. Brigans, R. Feenstra, and Gibson, MRS Symposia Proceedings 159 (Materials Research Society, Pittsburgh, 1990), p. 133.

${ }^{40}$ Wei Li, J.-S. Lin, M. Karimi, C. Moses, P. A. Dowben, and G. Vidali, Proceedings of the International Conference on the Structure of Surfaces, Milwaukee, 1990 (Springer-Verlag, Berlin, in press).

${ }^{41}$ G. Vidali, C. W. Hutchings, P. A. Dowben, M. Karimi, C. Moses, and M. Foresti, J. Vac. Sci. Technol. A 8, 3043 (1990).

${ }^{42}$ N. F. Mott, Proc. Phys. Soc. London Sect. A 62, 416 (1949).

${ }^{43}$ P. A. Dowben, Y. J. Kime, S. Varma, M. Onellion, and J. L. Erskine, Phys. Rev. B 36, 2519 (1987); P. A. Dowben, M. Onellion, and Y. J. Kime, Scanning Microsc. 2, 177 (1988).

${ }^{44}$ K. G. Eyink, B. C. Lamartine, W. V. Lampert, and T. W. Haas, Appl. Surf. Sci. 20, 215 (1985).

${ }^{45}$ Y. J. Kime, Jiandi Zhang, Ling Zhang, and P. A. Dowben (unpublished).

${ }^{46}$ P. A. Dowben, Y. J. Kime, D. LaGraffe, and M. Onellion, Surf. Interface Anal. 15, 163 (1990).

${ }^{47}$ S. Varma, Y. J. Kime, D. LaGraffe, P. A. Dowben, M. Onellion, and J. L. Erskine, J. Chem. Phys. 93, 2819 (1990).

${ }^{48}$ S. Svensson, N. Martensson, E. Basilier, P. A. Malquist, U. Gelius, and K. Siegbahn, J. Electron Spectrosc. Relat. Phenom. 9, 51 (1976).

${ }^{49}$ S. P. Shannon and K. Codling, J. Phys. B 11, 1193 (1978).

${ }^{50}$ P. H. Kobrin, P. A. Heimann, H. G. Kerkhoff, D. W. Lindle, C. M. Truesdale, T. A. Ferrett, U. Becker, and D. A. Shirley, Phys. Rev. A 27, 3031 (1983).

${ }^{51}$ S. Suzer, S. T. Lee, and D. A. Shirley, Phys. Rev. A 13, 1842 (1976).

52J. L. Dehnmer and J. Berkowitz, Phys. Rev. A 6, 484 (1974).

${ }^{53}$ G. van der Laan and B. T. Thole, Phys. Rev. Lett. 60, 1977 (1988).

${ }^{54}$ S. Asano, Y. Ishida, and F. Yonezawa, J. Phys. (Paris) Colloq. 41, C4-81 (1980); L. F. Mattheiss and W. W. Warren, Jr., Phys. Rev. B 16, 624 (1977).

${ }^{55}$ M. A. Mueller, T. Miller, and T.-C. Chiang, Phys. Rev. B 41, 5214 (1990).

${ }^{56}$ N. F. Mott, Philos. Mag. 13, 989 (1966).

${ }^{57}$ N. F. Mott, Philos. Mag. 26, 505 (1972).

${ }^{58}$ N. S. Hush and S. Suzer, Chem. Phys. Lett. 46, 411 (1977).

${ }^{59}$ S. Varma, Y. J. Kime, P. A. Dowben, M. Onellion, and J. L. Erskine, Phys. Lett. A 116, 66 (1986).

${ }^{60}$ P. A. Dowben, M. Onellion, S. Varma, Y. J. Kime, and J. L. Erskine, J. Vac. Sci. Technol. A 7, 2070 (1989); M. Onellion, P. A. Dowben, and J. L. Erskine, Phys. Lett. A 130, 171 (1988).

${ }^{61}$ F. Keller and F. Combet-Farnoux, J. Phys. B 12, 2821 (1979) and references cited therein.

${ }^{62}$ W. R. Johnson, V. Radojevic, Pranawa Deshmukh, and K. T. Cheng, Phys. Rev. A 25, 337 (1982).

${ }^{63}$ G. Broden, S. B. M. Hagstrom, and C. Norris, Phys. Kondens.
Mater. 15, 327 (1973).

${ }^{64}$ L. Ley, N. Martensson, and J. Azouly, Phys. Rev. Lett. 45, 1516 (1980).

${ }^{65}$ M. Salmeron, S. Ferrer, M. Jazzer, and G. A. Somorjai, Phys. Rev. B 28, 1158 (1983); 28, 6758 (1983).

${ }^{66}$ W. F. Egelhoff, Jr., Surf. Sci. Rep. 6, 253 (1987).

${ }^{67}$ P. H. Citrin and G. K. Wertheim, Phys. Rev. B 27, 3176 (1983).

${ }^{68}$ T. Hanyu, T. Miyahara, T. Komada, K.-I. Asada, H. Ohkuma, H. Ishii, K. Naito, H. Kato, and S. Yamaguchi, J. Phys. Soc. Jpn. 53, 3667 (1984).

${ }^{69}$ J. Kanski and P. O. Nilsson, J. Phys. F 11, 1859 (1981).

${ }^{70}$ J. F. Herbst, Phys. Rev. B 22, 4600 (1980).

${ }^{71}$ F. Riele, Jpn. J. Appl. Phys. 17, 314 (1978).

72J. P. Connerade, M. W. D. Mansfield, G. H. Newsom, D. H. Tracy, M. A. Baig, and K. Thimm, Philos. Trans. R. Soc. London Ser. A 290, 327 (1979); S. J. Rose, I. P. Grant, and J. P. Connerade, ibid. 296, 527 (1980), and references cited therein.

${ }^{73}$ M. A. Baig, J. P. Connerade, C. Mayhew, and K. Summer, J. Phys. B 17, 371 (1984).

${ }^{74}$ J. P. Connerade and M. A. P. Martin, J. Phys. B 13, L373 (1980).

${ }^{75}$ R. A. Rosenberg, M. G. White, G. Thornton, and D. A. Shirley, Phys. Rev. Lett. 43, 1384 (1979).

${ }^{76}$ P. H. Korbrin, R. A. Rosenberg, U. Becker, S. Southworth, C. M. Trusedale, D. W. Lindle, G. Thornton, M. G. White, E. D. Poliakoff, and D. A. Shirley, J. Phys. B 16, 4339 (1983).

${ }^{77}$ Photoemission in Solids I, Vol. 26 of Topics in Applied Physics, edited by M. Cardona and L. Ley (Springer-Verlag, Berlin, 1978).

${ }^{78}$ Y. Sakisaka, T. N. Rhodin, and P. A. Dowben, Solid State Commun. 49, 563 (1984).

${ }^{79}$ S. T. Manson, in Photoemission in Solids I, Vol. 26 of Topics in Applied Physics, edited by M. Cardona and L. Ley (SpringerVerlag, Berlin, 1978), pp. 135-164; H. Chacham, X. Phu, and S. G. Louie, Europhys. Lett. 14, 65 (1991).

${ }^{80}$ N. J. DiNardo, T. Maeda Wong, and E. W. Plummer, Phys. Rev. Lett. 65, 2177 (1990).

${ }^{81}$ K. Rademann, B. Kaiser, U. Even, and F. Hensel, Phys. Rev. Lett. 59, 2319 (1987).

${ }^{82}$ K. Rademann, B. Kaiser, T. Rech, and F. Hensel, Z. Phys. D 12, 431 (1989).

${ }^{83}$ K. Rademann, Ber. Bunsenges. Phys. Chem. 93, 653 (1989).

${ }^{84}$ G. M. Pastor, P. Sampfli, and K. H. Bennemann, Europhys. Lett. 7, 419 (1988).

${ }^{85}$ These lattice constants are tabulated from a number of different calculations in Ref. 1. The original references are $\mathbf{H}$. Overhof, H. Uchtmann, and F. Hensel, J. Phys. F 6, 523 (1976); P. Fritzson and K. F. Berggsen, Solid State Commun. 19, 385 (1976); M. A. C. Devillers and R. G. Ross, J. Phys. F 5, 73 (1975); F. Yonezawa, Y. Ishida, F. Martino, and S. Asano, in Liquid Metals, edited by R. Evans and D. A. Greenwood (I.O.P., Bristol, 1977), pp. 385; and Ref. 54.

${ }^{86}$ R. G. Jones and D. L. Perry, Surf. Sci. 71, 59 (1979); 82, 540 (1979).

${ }^{87}$ W. F. Egelhoff, Jr., D. L. Perry, and J. W. Linnett, Surf. Sci. 54, 670 (1976). 